\title{
On Distance-Regular Graphs with Height Two
}

\author{
MASATO TOMIYAMA \\ Graduate School of Mathematics, Kyushu University, Higashi-ku, Fukuoka 812, Japan
}

Received February 25, 1994; Revised January 6, 1995

\begin{abstract}
Let $\Gamma$ be a distance-regular graph with diameter at least three and height $h=2$, where $h=\max \left\{i: p_{d !}^{d}\right.$ $\neq 0\}$. Suppose that for every $\alpha$ in $\Gamma$ and $\beta$ in $\Gamma_{d}(\alpha)$, the induced subgraph on $\Gamma_{d}(\alpha) \cap \Gamma_{2}(\beta)$ is a clique. Then $\Gamma$ is isomorphic to the Johnson graph $J(8,3)$.
\end{abstract}

Keywords: distance-regular graph, strongly regular graph, height, clique, Johnson graph

\section{Introduction}

Let $\Gamma$ be a connected undirected simple finite graph. We identify $\Gamma$ with the set of vertices. For vertices $u$ and $v$, let $\partial(u, v)$ denote the distance between $u$ and $v$, i.e. the length of a shortest path from $u$ to $v$ in $\Gamma$. Let $d=d(\Gamma)$ denote the diameter of $\Gamma$, i.e. the maximal distance of two vertices in $\Gamma$. We set

$$
\Gamma_{l}(u)=\{x \in \Gamma: \partial(u, x)=i\} \quad(0 \leq i \leq d) .
$$

$\Gamma$ is said to be distance-regular if the cardinality of the set $\Gamma_{t}(u) \cap \Gamma_{J}(v)$ depends only on the distance between $u$ and $v$. In this case we write

$$
p_{i j}^{l}=\left|\Gamma_{i}(u) \cap \Gamma_{j}(v)\right| \quad(0 \leq i, j, l \leq d),
$$

where $l=\partial(u, v)$. Let

$$
k_{i}=p_{u l}^{0}=\left|\Gamma_{1}(u)\right| \quad(0 \leq i \leq d) .
$$

In particular $k=k_{1}$ is the valency of $\Gamma$. Let

$$
c_{i}=p_{1 i-1}^{i}, \quad a_{i}=p_{1_{l}}^{i}, \quad b_{l}=p_{1_{l+1}}^{i} \quad(0 \leq i \leq d) .
$$

They are called the intersection numbers of $\Gamma$, and

$$
\iota(\Gamma)=\left\{\begin{array}{cccccccc}
* & c_{1} & c_{2} & \cdots & c_{t} & \cdots & c_{d-1} & c_{d} \\
a_{0} & a_{1} & a_{2} & \cdots & a_{1} & \cdots & a_{d-1} & a_{d} \\
b_{0} & b_{1} & b_{2} & \cdots & b_{i} & \cdots & b_{d-1} & *
\end{array}\right\}
$$

is called the intersection array of $\Gamma$.

The following are basic properties of intersection numbers, which we use implicitly in this paper.

(1) $c_{\imath}+a_{i}+b_{t}=k \quad(0 \leq i \leq d)$,

(2) $1=c_{1} \leq c_{2} \leq c_{3} \leq \cdots \leq c_{d-1} \leq c_{d} \leq k$, 
(3) $k=b_{0}>b_{1} \geq b_{2} \geq \cdots \geq b_{d-2} \geq b_{d-1} \geq 1$,

(4) $k_{i} b_{i}=k_{i+1} c_{i+1} \quad(0 \leq i \leq d-1)$,

(5) $k_{l} p_{i j}^{l}=k_{i} p_{l j}^{i}=k_{j} p_{l i}^{j} \quad(0 \leq i, j, l \leq d)$,

(6) $p_{i j}^{l} \neq 0$ if $l=i+j$ or $l=|i-j|$,

(7) $c_{i} \leq b_{j}$ if $i+j \leq d$.

A graph is said to be strongly regular if it is distance-regular with diameter 2 .

A graph is called a clique when any two of its vertices are adjacent. A coclique is a graph in which no two vertices are adjacent.

Information about the general theory of distance-regular graphs is given in [1], [3] and [5].

Let $X$ be a finite set of cardinality $v$ and $V=\{T \subset X:|T|=e\}$. The Johnson graph $J(v, e)$ is a graph whose vertex set is $V$ and two vertices $x$ and $y$ are adjacent if and only if $|x \cap y|=e-1$. It is well known that $J(v, e)$ is a distance-regular graph.

In this paper we identify a subset $A$ of $\Gamma$ with the induced subgraph on $A$ and define the following terminology.

A subgraph $A$ of $\Gamma$ is called geodetically closed if for all vertices $x$ and $y$ in $A$ with $\partial(x, y)=i, \Gamma_{i-1}(x) \cap \Gamma_{1}(y)$ is in $A$. For subsets $A$ and $B$ of $\Gamma$, let $\partial(A, B)=\min (\partial(x, y)$ : $x \in A, y \in B\}$. Let $h=\max \left\{i: p_{d i}^{d} \neq 0\right\}$ be the height of $\Gamma$.

A distance-regular graph $\Gamma$ is of height 0 if and only if $\Gamma$ is an antipodal 2-cover, and is of height 1 if and only if $\Gamma_{d}(\alpha)$ is a clique for every $\alpha$ in $\Gamma$. So if the height of $\Gamma$ is $1, \Gamma$ is the distance- 2 graph of a generalized odd graph (see Proposition 4.2.10 of [5]). This paper is concerned with a distance-regular graph of height 2 .

Theorem 1.1 Let $\Gamma$ be a distance-regular graph with diameter $d$ at least 3 and height $h=2$. Suppose that for every $\alpha$ in $\Gamma$ and $\beta$ in $\Gamma_{d}(\alpha), \Gamma_{d}(\alpha) \cap \Gamma_{2}(\beta)$ is a clique. Then $d=3$ and $\Gamma$ is isomorphic to $J(8,3)$.

In [8] and [9] H. Suzuki showed that $d(\Gamma)$ is bounded by a function depending only on $k_{d}$ if $\Gamma_{d}(\alpha)$ is not isomorphic to a coclique. Hence if $\Gamma_{d}(\alpha)$ is isomorphic to a given strongly regular graph $\Delta$, then there are only finitely many possibilities for $\Gamma$.

On the other hand if $\Gamma$ is isomorphic to Hamming graphs $H(2, q)(q \geq 3)$, Johnson graphs $J(v, 2)(v \geq 6)$ or $J(2 d+2, d)(d \geq 2)$, then $\Gamma_{d}(\alpha)$ is isomorphic to a strongly regular graph.

Is it possible to characterize these distance-regular graphs by the antipodal structures $\Gamma_{d}(\alpha)$ ?

Let $\Delta$ be a graph with diameter 2. Suppose $\Gamma_{d}(\alpha)$ is isomorphic to $\Delta$ for every $\alpha$ in $\Gamma$. Then the height of $\Gamma$ becomes 2 . It is easy to see that in this situation $\Delta$ is distance-degree regular, i.e. $\left|\Delta_{1}(\beta)\right|=p_{d 1}^{d},\left|\Delta_{2}(\beta)\right|=p_{d 2}^{d}$ do not depend on the choice of $\beta$ in $\Delta$.

Let $\Delta$ be a distance-degree regular graph with diameter 2 such that $\Delta_{2}(\beta)$ is a clique for every $\beta$ in $\Delta$. The theorem above shows that if there exists a distance-regular graph $\Gamma$ of diameter $d$ at least 3 such that $\Gamma_{d}(\alpha)$ is isomorphic to $\Delta$ for every $\alpha$ in $\Gamma$, then $\Gamma$ is isomorphic to $J(8,3)$ and $\Delta$ is isomorphic to $J(5,2)$.

We note that there are many distance-degree regular graphs of diameter 2 such that $\Delta_{2}(\beta)$ is a clique for every $\beta$ in $\Delta$. The complete bipartite graphs $K_{s, s}$, the pentagon and the complements of strongly regular graphs with $a_{1}=0$ are in this class.

It is not hard to construct graphs in this class which are not strongly regular. For example, a clique extension $\Delta$ of a graph $\Lambda$ in this class is also in it. By a clique extension we mean 
the following. Let $K^{u}(u \in \Lambda)$ be finite disjoint sets of the same size. $\Delta$ is a graph whose vertex set is $\cup_{u \in \Lambda} K^{u}$ and two distinct vertices $x \in K^{u}$ and $y \in K^{v}$ are adjacent if and only if $u=v$ or $u$ and $v$ are adjacent in $\Lambda$.

Corollary 1.2 Let $\Gamma$ be a distance-regular graph with diameter $d$ at least 3 , and $\Delta$ a strongly regular graph such that $\Delta_{2}(\beta)$ is a disjoint union of cliques for every $\beta$ in $\Delta$. If $\Gamma_{d}(\alpha)$ is isomorphic to $\Delta$ for every $\alpha$ in $\Gamma$, then $d=3$ and $\Gamma$ is isomorphic to $J(8,3)$.

Proof: Suppose $\Delta_{2}(\beta)$ is not a clique. Then it follows from Lemma 3.1 of [6] that $\Delta$ is a complete multipartite graph $K_{\mathrm{r} \times s}$. Then by an unpublished work of A. Hiraki and $\mathrm{H}$. Suzuki (see Appendix), we get $d \leq 2$. So we may assume that $\Delta_{2}(\beta)$ is a clique. Now the assertion follows from Theorem 1.1.

\section{Intersection diagram}

In this section we shall introduce the intersection diagrams of rank $d$ which we use as our main tool.

Let $\alpha, \beta \in \Gamma$ with $\partial(\alpha, \beta)=d$. Set

$$
D_{j}^{i}=D_{j}^{i}(\alpha, \beta)=\Gamma_{i}(\alpha) \cap \Gamma_{j}(\beta) \quad(0 \leq i, j \leq d) .
$$

It is easy to see the following.

(1) $D_{j}^{i}=\phi$ if $d>i+j$,

(2) $D_{d-i}^{i} \neq \phi$ if $0 \leq i \leq d$,

(3) There is no edge between $D_{j}^{i}$ and $D_{g}^{f}$ if $|i-f|>1$ or $|j-g|>1$.

An intersection diagram of rank $d$ with respect to $(\alpha, \beta)$ is the collection $\left\{D_{j}^{\imath}\right\}_{l, j}$ with lines between $D_{j}^{i}$ 's and $D_{g}^{f}$ 's. We draw a line

$$
D_{j}^{\prime}-D_{g}^{f}
$$

if there is possibility of existence of edges between $D_{j}^{i}$ and $D_{g}^{f}$, and we erase the line when we know there is no edge between $D^{i}$, and $D_{g}^{f}$.

In the following $e(A, B)$ denotes the number of edges between subsets $A$ and $B$ of $\Gamma$, and $e(\{\gamma\}, A)=e(\gamma, A)$. We write $\alpha \sim \beta$, when $\beta$ is in $\Gamma_{1}(\alpha)$, and $\alpha \neq \beta$, otherwise.

The following are straightforward and useful for determining the form of the intersection diagram.

For each $\gamma \in D_{j}^{i}$, we have the following.

(4) $c_{i}=e\left(\gamma, D_{j+1}^{i-1}\right)+e\left(\gamma, D_{j}^{i-1}\right)+e\left(\gamma, D_{j-1}^{i-1}\right)$,

$c_{j}=e\left(\gamma, D_{j-1}^{i+1}\right)+e\left(\gamma, D_{j-1}^{i}\right)+e\left(\gamma, D_{j-1}^{i-1}\right)$,

(5) $a_{i}=e\left(\gamma, D_{J+1}^{i}\right)+e\left(\gamma, D_{j}^{i}\right)+e\left(\gamma, D_{J-1}^{i}\right)$,

$a_{3}=e\left(\gamma, D_{j}^{i+1}\right)+e\left(\gamma, D_{j}^{i}\right)+e\left(\gamma, D_{j}^{i-1}\right)$,

(6) $b_{t}=e\left(\gamma, D_{j+1}^{i+1}\right)+e\left(\gamma, D_{j}^{i+1}\right)+e\left(\gamma, D_{j-1}^{i+1}\right)$,

$b_{\jmath}=e\left(\gamma, D_{\jmath+1}^{i+1}\right)+e\left(\gamma, D_{j+1}^{i}\right)+e\left(\gamma, D_{\jmath+1}^{i-1}\right)$. 


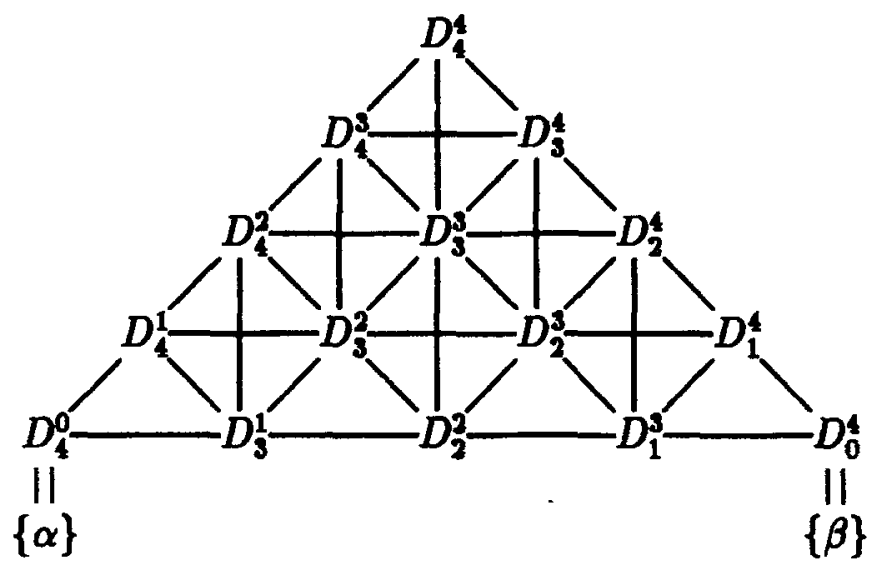

Figure 1.

Figure 1 is an example of the intersection diagram of rank $d=d(\Gamma)$ with $d=4$.

For the properties and applications of intersection diagrams, see for example [2] and [4].

\section{Preliminaries}

In this section we determine the shape of the intersection diagram under the hypothesis of Theorem 1.1, and prove some basic lemmas.

Suppose there is a vertex $x \in D_{j}^{i}$, for some $i, j$ with $i \geq 3, j \geq 3, i+j \geq d+3$. Then there is a vertex $y \in \Gamma_{d}(\alpha) \cap \Gamma_{d-i}(x)$. Since $\beta, y \in \Gamma_{d}(\alpha)$ and the height $h=2$,

On the other hand,

$$
\partial(\beta, y) \leq 2 .
$$

$$
\partial(\beta, y) \geq|\partial(\beta, x)-\partial(x, y)|=|(i+j)-d| \geq 3,
$$

which is impossible. So

$$
D_{j}^{i}=\emptyset \quad \text { for } i \geq 3, j \geq 3, i+j \geq d+3 .
$$

Therefore the intersection diagram becomes as in Fig. 2 .

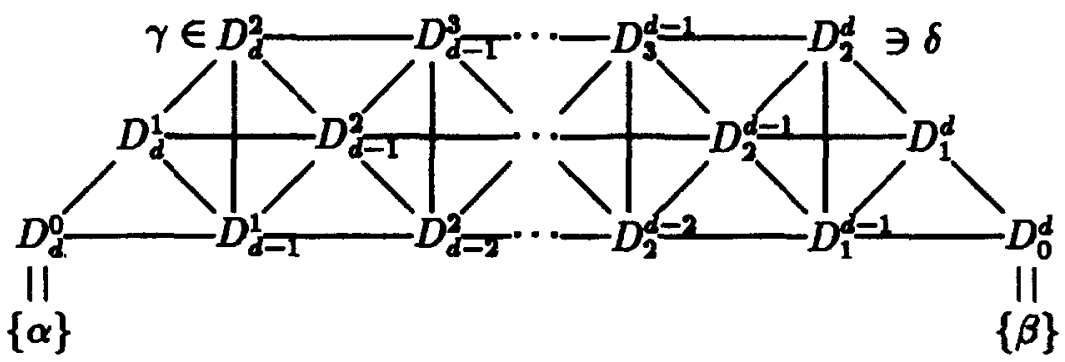


Take any $\gamma \in D_{d}^{2}$, then

$$
\Gamma_{l}(\alpha) \cap \Gamma_{i-2}(\gamma) \subseteq D_{d-i+2}^{i} \quad \text { for } 2 \leq i \leq d .
$$

Since $p_{l i-2}^{2} \neq 0$, we get

$$
D_{d-i+2}^{i} \neq \phi \text {, i.e. } p_{t d-i+2}^{d} \neq 0 \quad \text { for } 2 \leq i \leq d .
$$

Since $k_{l} p_{d d-i+2}^{l}=k_{d} p_{i d-l+2}^{d} \neq 0$, we have

$$
p_{d d-i+2}^{i} \neq 0 \text { for } 2 \leq i \leq d .
$$

Let $\kappa_{1}=p_{d 1}^{d}=a_{d}$ and $\kappa_{2}=p_{d 2}^{d}$. Then $k_{d}=1+\kappa_{1}+\kappa_{2}$. Since $D_{2}^{d}$ is a clique, for any $\delta \in D_{2}^{d}, e\left(\delta, D_{2}^{d}\right)=\kappa_{2}-1$.

Lemma 3.1 For every $\alpha$ in $\Gamma$ and every $\beta, \gamma, \delta$ in $\Gamma_{d}(\alpha), \partial(\beta, \gamma)+\partial(\gamma, \delta)+\partial(\delta, \beta) \leq 5$.

Proof: Suppose there are vertices $\beta, \gamma, \delta \in \Gamma_{d}(\alpha)$ such that $\partial(\beta, \gamma)+\partial(\gamma, \delta)+\partial(\delta, \beta) \geq 6$. Since the height $h=2$,

$$
\partial(\beta, \gamma)=\partial(\gamma, \delta)=\partial(\delta, \beta)=2
$$

So $\gamma, \delta \in D_{2}^{d}$. This contradicts that $D_{2}^{d}$ is a clique.

Lemma $3.2 \partial\left(D_{d-2}^{2}, D_{2}^{d}\right) \geq d-1$.

Proof: Suppose there are vertices $u \in D_{d-2}^{2}$ and $v \in D_{2}^{d}$ such that $\partial(u, v) \leq d-2$ (see Fig. 3).

We can take $w \in \Gamma_{d}(\alpha) \cap \Gamma_{d}(u)$ because $p_{d d}^{2} \neq 0$. Since $\beta, v, w \in \Gamma_{d}(\alpha)$ with $\partial(\beta, v)=2$, by Lemma 3.1, we have $\partial(w, \beta)=1$ or $\partial(w, v)=1$. Since $\partial(u, \beta)=d-2$ and $\partial(u, v) \leq d-2$, we get $\partial(u, w) \leq d-1$. This contradicts $w \in \Gamma_{d}(u)$.

Lemma $3.3 e\left(D_{d-l-1}^{t+1}, D_{d-l}^{i+2}\right)=0$ for $0 \leq i \leq d-2$.

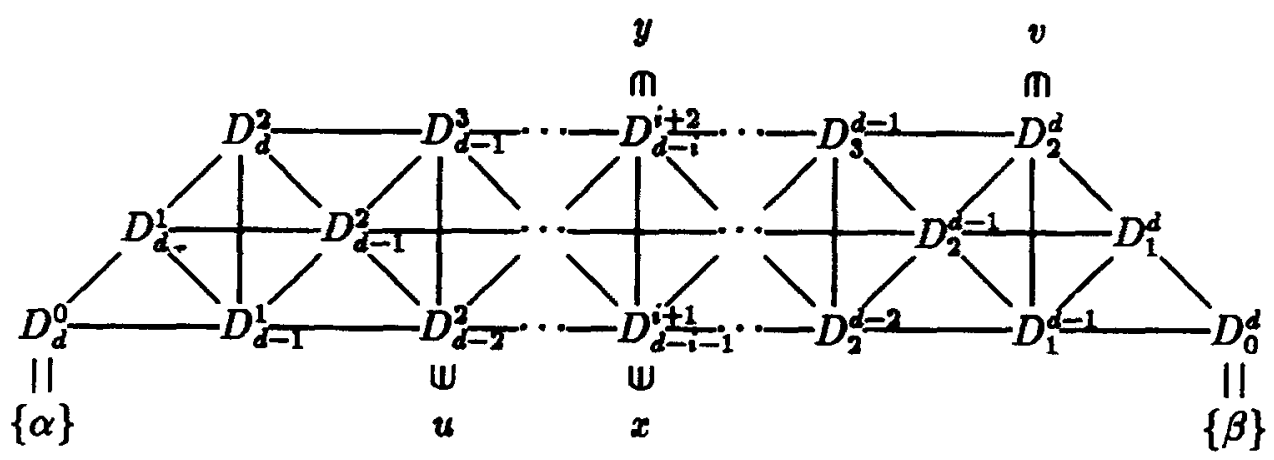

Figure 3. 


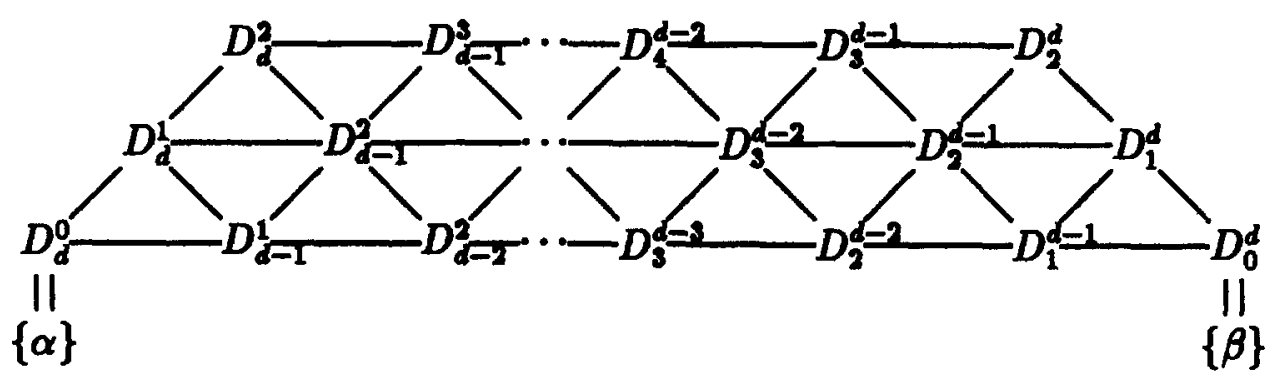

Figure 4 .

Proof: Suppose not. Then there is an edge $x \sim y$ such that $x \in D_{d-i-1}^{i+1}, y \in D_{d-i}^{i+2}$. If $i \geq 1$, we can take $u \in D_{d-2}^{2}$ with $\partial(u, x)=i-1$ and $v \in D_{2}^{d}$ with $\partial(y, v)=d-i-2$ (see Fig. 3). We get $\partial(u, v)=d-2$, which contradicts Lemma 3.2. Since $e\left(D_{1}^{d-1}, D_{2}^{d}\right)=0$, we get $e\left(D_{d-1}^{1}, D_{d}^{2}\right)=0$ by symmetry.

By Lemma 3.3, the intersection diagram becomes as in Fig. 4.

Lemma 3.4 The following hold.

(1) $\Gamma_{d}(\alpha)$ is geodetically closed for every $\alpha$ in $\Gamma$,

(2) $\kappa_{1} \geq 2$,

(3) $c_{2}=\kappa_{1}-\kappa_{2}+1$.

\section{Proof:}

(1) Let $\beta, \gamma \in \Gamma_{d}(\alpha)$ with $\partial(\beta, \gamma)=i$. Since the height $h=2$, we only consider the case $i=2$. Then $\gamma \in D_{2}^{d}$. Since $e\left(D_{1}^{d-1}, D_{2}^{d}\right)=0$,

$$
\Gamma_{1}(\beta) \cap \Gamma_{1}(\gamma) \subseteq D_{1}^{d} \subseteq \Gamma_{d}(\alpha) .
$$

(2) For any $\gamma \in D_{2}^{d}$, there is $\delta \in D_{1}^{d}$ such that $\gamma \sim \delta \sim \beta$. So

$$
\kappa_{1}=p_{d 1}^{d}=\left|\Gamma_{d}(\alpha) \cap \Gamma_{1}(\delta)\right| \geq 2 .
$$

(3) Take $\gamma \in D_{2}^{d}$, then $\kappa_{1}=a_{d}=e\left(\gamma, D_{2}^{d}\right)+e\left(\gamma, D_{1}^{d}\right)=\kappa_{2}-1+e\left(\gamma, D_{1}^{d}\right)$. From Lemma 3.3, we get

$$
c_{2}=e\left(\gamma, D_{1}^{d}\right)=\kappa_{1}-\kappa_{2}+1
$$

Lemma 3.5 $c_{3} \neq 1$.

Proof: Suppose $c_{3}=1$. Then for any $x \in D_{3}^{d-1}$,

$$
b_{d-1}=e\left(x, D_{2}^{d}\right) \leq e\left(x, D_{2}^{d}\right)+e\left(x, D_{2}^{d-1}\right)=c_{3}=1 .
$$

Hence we have

$$
b_{d-1}=1 \text {. }
$$




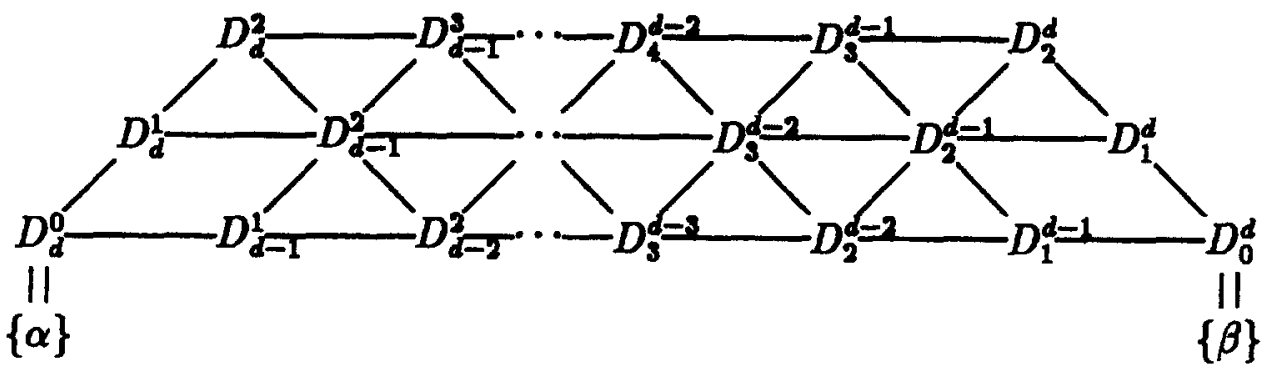

Figure 5 .

For any $y \in D_{1}^{d-1}$,

$$
1=b_{d-1}=e\left(y, D_{1}^{d}\right)+e\left(y, D_{0}^{d}\right)=e\left(y, D_{1}^{d}\right)+1 .
$$

So we get $e\left(y, D_{1}^{d}\right)=0$. Hence we have

$$
e\left(D_{1}^{d-1}, D_{1}^{d}\right)=0 .
$$

Therefore the intersection diagram becomes as in Fig. 5 .

For any $\gamma \in D_{2}^{d}$ and any $\delta \in D_{1}^{d}$, we get

$$
e\left(\gamma, D_{1}^{d}\right)=c_{2}, e\left(\delta, D_{1}^{d}\right)=a_{1} .
$$

So for any two vertices $u, v \in \Gamma_{d}(\alpha)$, the number of vertices which are adjacent to $u$ and $v$ in $\Gamma_{d}(\alpha)$ is $c_{2}$ if $u \not \gamma v$ and $a_{1}$ if $u \sim v$. Hence $\Gamma_{d}(\alpha)$ becomes strongly regular.

We use bar to distinguish the parameters of $\Delta=\Gamma_{d}(\alpha)$ from those of $\Gamma$. Then $\bar{k}=\kappa_{1}$, $\overrightarrow{k_{2}}=\kappa_{2}$.

Since $c_{3}=c_{2}=1$, Lemma 3.4(3) implies that $\kappa_{1}=\kappa_{2}$. Hence the intersection array of $\Delta$ becomes

$$
\iota(\Delta)=\left\{\begin{array}{ccc}
* & 1 & 1 \\
0 & \kappa_{1}-2 & \kappa_{1}-1 \\
\kappa_{1} & 1 & *
\end{array}\right\} .
$$

Since $\overline{b_{1}}=\overline{c_{2}}=1$, we get $\overline{a_{1}}=0$ (see Proposition 5.5.1 of [5]). So we have $\kappa_{1}=2$ and

$$
\begin{aligned}
k p_{d d}^{1} & =k_{d} p_{d 1}^{d}=10, \\
k_{2} p_{d d}^{2} & =k_{d} p_{d 2}^{d}=10 .
\end{aligned}
$$

As $k<k_{2}$ (see Lemma 5.1.2 of [5]),

$$
k=5, \quad k_{2}=10 .
$$

So we have

$$
b_{1}=2, \quad a_{1}=2 .
$$

Hence $\Gamma$ is locally pentagon and we know $\Gamma$ is isomorphic to the icosahedron (see Proposition 1.1.4 of [5]). This contradicts $k_{2}=10$. 


\section{The case $d \geq 4$}

In this section we discuss the case $d \geq 4$ and prove this case does not occur.

Lemma 4.1 Suppose $d \geq 4$. Then the following hold.

(1) $b_{2} \geq c_{d-1}$,

(2) $b_{d-2} \geq c_{3}$.

\section{Proof:}

(1) Take $\gamma \in D_{2}^{d}$, then

$$
b_{2}=e\left(\gamma, D_{3}^{d-1}\right) \leq e\left(\gamma, D_{3}^{d-1}\right)+e\left(\gamma, D_{2}^{d-1}\right)=c_{d} .
$$

Suppose $b_{2}=c_{d}$, then $b_{2}=c_{d} \geq c_{d-1}$. So we may assume $b_{2}<c_{d}$. Then $e\left(\gamma, D_{2}^{d-1}\right) \neq 0$, so there is $\delta \in D_{2}^{d-1}$ such that $\gamma \sim \delta$ (see Fig. 6).

Claim $e\left(\delta, D_{2}^{d-2}\right)=0$.

Suppose for some $x \in D_{2}^{d-2}$ such that $x \sim \delta$. Since there is $y \in D_{d-2}^{2}$ such that $\partial(y, x)=d-4$, we get $\partial(y, \gamma)=d-2$. This contradicts Lemma 3.2. Hence we get $e\left(\delta, D_{2}^{d-2}\right)=0$.

By Claim, we get

$$
b_{2}=e\left(\delta, D_{3}^{d-2}\right)+e\left(\delta, D_{3}^{d-1}\right) \geq e\left(\delta, D_{3}^{d-2}\right)=c_{d-1} .
$$

(2) Take $u \in D_{d-2}^{4}$ and argue similarly as in (1).

Lemma 4.2 Suppose $d \geq 4$. Then for every $x$ in $D_{d-2}^{2}$, there are $\gamma$ and $\delta$ in $\Gamma_{d}(x)$ such that $\gamma$ in $D_{2}^{d}$ and $\delta$ in $D_{4}^{d-2}$.

Proof: Since $p_{d d}^{2} \neq 0$, take $\gamma \in \Gamma_{d}(\alpha) \cap \Gamma_{d}(x)$. Then $\partial(\beta, \gamma) \geq \partial(x, \gamma)-\partial(x, \beta)=2$. $\beta, \gamma \in \Gamma_{d}(\alpha)$ and the height $h=2$, so $\partial(\beta, \gamma)=2$. Hence we get

$$
\gamma \in D_{2}^{d} \text {. }
$$

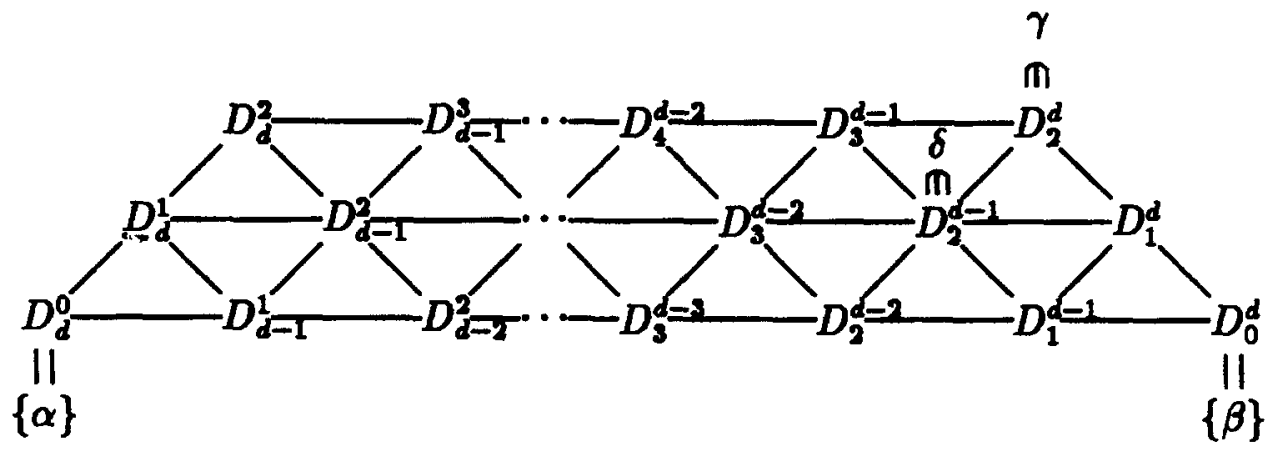

Figure 6. 


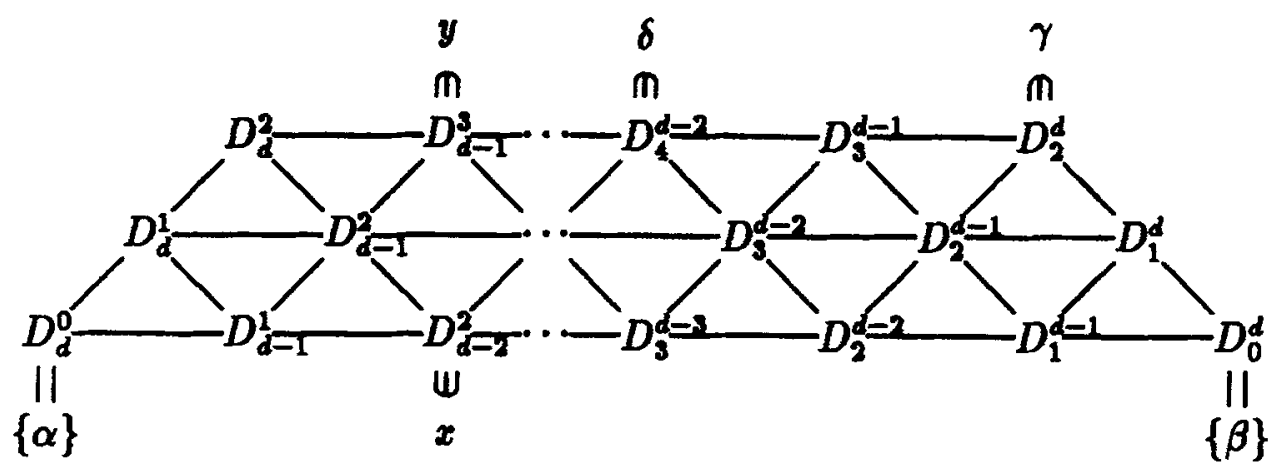

Figure 7

Since $p_{d 4}^{d-2} \neq 0$, take $\delta \in \Gamma_{d}(x) \cap \Gamma_{4}(\beta)$. Then $\partial(\alpha, \delta) \geq \partial(x, \delta)-\partial(\alpha, x)=d-2$. Since $D_{4}^{l}=\phi$ for $i \geq d-1$, we get

$$
\delta \in D_{4}^{d-2}
$$

Lemma 4.3 Suppose $d \geq 4$. Then $\partial\left(D_{d-2}^{2}, D_{d-1}^{3}\right) \geq 3$.

Proof: Suppose there are $x \in D_{d-2}^{2}, y \in D_{d-1}^{3}$ such that $\partial(x, y)=2$. Then there is $z \in \Gamma_{d}(x) \cap \Gamma_{d}(y)$. By Lemma 4.2, there are $\gamma, \delta \in \Gamma_{d}(x)$ such that $\gamma \in D_{2}^{d}, \delta \in D_{4}^{d-2}$ (see Fig. 7). Since $\gamma, \delta, z \in \Gamma_{d}(x)$ with $\partial(\gamma, \delta)=2$, Lemma 3.1 implies that $\partial(z, \gamma) \leq 1$ or $\partial(z, \delta) \leq 1$.

Case 1. $\partial(z, \gamma) \leq 1$.

Since there is $u \in D_{2}^{d}$ such that $\partial(y, u)=d-3$ and $D_{2}^{d}$ is a clique, $\partial(y, \gamma) \leq d-2$. So we get $\partial(y, z) \leq d-1$, which contradicts $z \in \Gamma_{d}(y)$.

Case 2. $\partial(z, \delta) \leq 1$.

There is $v \in D_{d}^{2}$ such that $\partial(\delta, v)=d-4$ and there is $w \in D_{d}^{2}$ such that $\partial(y, w)=1$. As $D_{d}^{2}$ is a clique, $\partial(y, z) \leq d-1$. This is a contradiction.

\section{Lemma $4.4 d=3$.}

Proof: Suppose $d \geq 4$. Take $x \in D_{d-2}^{2}$. If $b_{d-2}>c_{2}$, then we can take an edge $x \sim z$ such that $z \in D_{d-1}^{2}$. By Lemma 4.1(1) $b_{2} \geq c_{d-1}$. So

$$
\begin{aligned}
& e\left(z, D_{d-1}^{3}\right)+e\left(z, D_{d-2}^{3}\right) \geq e\left(z, D_{d-2}^{3}\right)+e\left(z, D_{d-2}^{2}\right), \\
& \quad e\left(z, D_{d-1}^{3}\right) \geq e\left(z, D_{d-2}^{2}\right) \geq e(z, x)=1 .
\end{aligned}
$$

Hence we can take an edge $z \sim y$ such that $y \in D_{d-1}^{3}$. So $\partial(x, y)=2$, which contradicts Lemma 4.3. We may assume $b_{d-2}=c_{2}$. By Lemma 4.1 (2), $c_{2}=b_{d-2} \geq c_{3}$. Therefore from Theorem 5.4.1 of [5] we get $c_{3}=1$. This contradicts Lemma 3.5. 


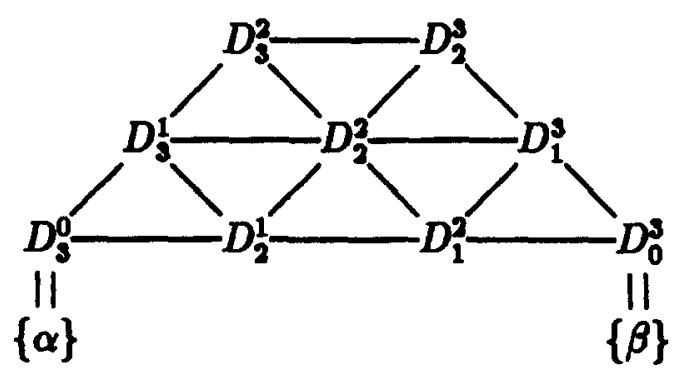

Figure 8.

\section{Proof of Theorem 1.1}

In the following we may assume $d=3$. The intersection diagram becomes as in Fig. 8 .

Lemma 5.1 For every $\gamma$ in $D_{1}^{2}, \Gamma_{3}(\alpha) \cap \Gamma_{3}(\gamma) \subseteq D_{2}^{3}$. In particular $p_{33}^{2} \leq p_{32}^{3}$, and the equality holds if and only if $b_{2}=c_{3}$.

Proof: Take $\gamma \in D_{1}^{2}$. Since $\gamma \sim \beta$ and $D_{1}^{3} \subseteq \Gamma_{1}(\beta)$, we get

$$
\Gamma_{3}(\alpha) \cap \Gamma_{3}(\gamma) \subseteq D_{2}^{3}
$$

Therefore

$$
p_{33}^{2}=\left|\Gamma_{3}(\alpha) \cap \Gamma_{3}(\gamma)\right| \leq\left|D_{2}^{3}\right|=p_{32}^{3}
$$

Since $\frac{p_{32}^{3}}{p_{33}^{2}}=\frac{k_{2}}{k_{3}}=\frac{c_{3}}{b_{2}}$

$$
p_{33}^{2}=p_{32}^{3} \quad \text { if and only if } b_{2}=c_{3}
$$

Lemma 5.2 For every $x$ in $D_{3}^{2}, \Gamma_{3}(\alpha) \cap \Gamma_{1}(x)=D_{2}^{3}$. In particular $b_{2}=\kappa_{2}$.

Proof: For any $x \in D_{3}^{2}$,

$$
\Gamma_{3}(\alpha) \cap \Gamma_{1}(x) \subseteq D_{2}^{3}
$$

By way of contradiction, suppose there is $y \in D_{2}^{3}$ such that $x \not y$ (see Fig. 9).

Since $D_{2}^{3}$ is a clique,

$$
\Gamma_{3}(\alpha) \cap \Gamma_{1}(x) \subseteq \Gamma_{1}(y)
$$

So we know

$$
\partial(x, y)=2
$$

Take $z \in \Gamma_{3}(x) \cap \Gamma_{3}(y)$. Since the height $h=2, z \notin \Gamma_{3}(\alpha) \cup \Gamma_{3}(\beta)$. So $\partial(\alpha, z)=2$ or $\partial(\beta, z)=2$. We may assume

$$
\partial(\alpha, z)=2
$$




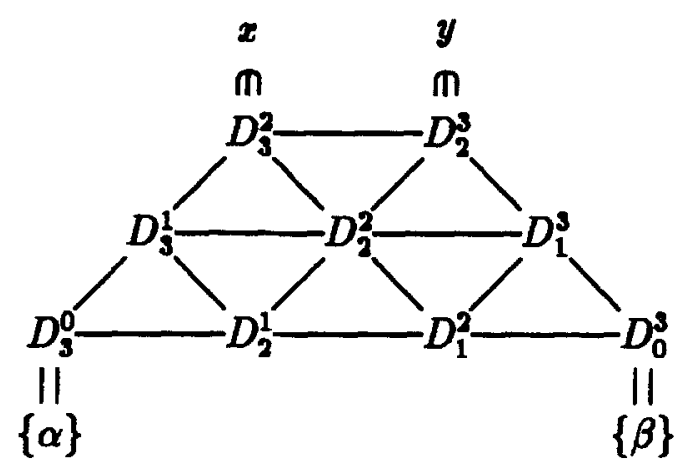

Figure 9.

From Lemma 3.4(1), $\Gamma_{3}(z)$ is geodetically closed. Since $x, y \in \Gamma_{3}(z)$ with $\partial(x, y)=2$ and $D_{2}^{3}$ is a clique,

$$
\begin{aligned}
\Gamma_{3}(z) & \supseteq\left(\Gamma_{1}(x) \cap \Gamma_{1}(y)\right) \cup\{y\} \cup\{x\} \\
& \supseteq\left(\Gamma_{3}(\alpha) \cap \Gamma_{1}(x)\right) \cup\{y\} .
\end{aligned}
$$

So

$$
\Gamma_{3}(\alpha) \cap \Gamma_{3}(z) \supseteq\left(\Gamma_{3}(\alpha) \cap \Gamma_{1}(x)\right) \cup\{y\}
$$

Claim $1 \quad b_{2}=c_{3}$.

Suppose there is some $\gamma \in D_{2}^{3}$ such that $\gamma \notin \Gamma_{3}(\alpha) \cap \Gamma_{3}(z)$. Then $\partial(z, \gamma)=2$ because $D_{2}^{3}$ is a clique and $\partial(z, y)=3$. So

$$
\begin{aligned}
\Gamma_{3}(z) \cap \Gamma_{1}(\gamma) & \supseteq\left(\left(\Gamma_{3}(\alpha) \cap \Gamma_{1}(x)\right) \cup\{y\}\right) \cap \Gamma_{1}(\gamma) \\
& =\left(\Gamma_{3}(\alpha) \cap \Gamma_{1}(x)\right) \cup\{y\} .
\end{aligned}
$$

In this case

$$
b_{2} \geq b_{2}+1
$$

which is impossible. Hence we get

$$
\Gamma_{3}(\alpha) \cap \Gamma_{3}(z) \supseteq D_{2}^{3} \quad \text { i.e. } p_{33}^{2} \geq p_{32}^{3} .
$$

From Lemma 5.1, we get

$$
b_{2}=c_{3}
$$

By Claim 1, for any $\delta \in D_{3}^{2}$,

$$
e\left(\delta, D_{2}^{3}\right)=b_{2}=c_{3}=e\left(\delta, D_{2}^{3}\right)+e\left(\delta, D_{2}^{2}\right) .
$$

So we get $e\left(\delta, D_{2}^{2}\right)=0$. Hence we have

$$
e\left(D_{3}^{2}, D_{2}^{2}\right)=0
$$




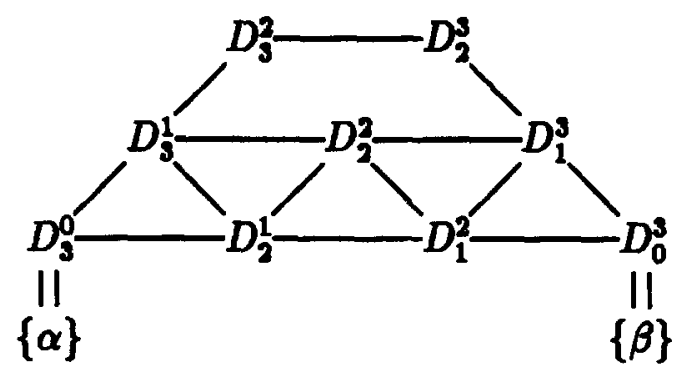

Figure 10.

Therefore the intersection diagram becomes as in Fig. 10.

Claim $2 D_{2}^{2} \neq \emptyset$.

Suppose $D_{2}^{2}=\emptyset$. Then for any $u \in D_{2}^{1}$,

$$
b_{1}=e\left(u, D_{1}^{2}\right)=c_{2} .
$$

By Claim 1, $c_{3}=b_{2} \leq b_{1}=c_{2}$. Hence, by Theorem 5.4 .1 of [5], we get $c_{3}=1$. This contradicts Lemma 3.5.

By Claim 2, take $\epsilon \in D_{2}^{2}$, then

$$
c_{3}=b_{2}=e\left(\epsilon, D_{1}^{3}\right) \leq e\left(\epsilon, D_{1}^{3}\right)+e\left(\epsilon, D_{1}^{2}\right)=c_{2} .
$$

Hence by Theorem 5.4.1 of [5], we get

$$
c_{3}=1 .
$$

This contradicts Lemma 3.5. Therefore we get

$$
\Gamma_{3}(\alpha) \cap \Gamma_{1}(x)=D_{2}^{3} .
$$

Lemma 5.3 $2 p_{33}^{1}=\kappa_{1}+p_{33}^{2}+1$.

Proof: Take any $x \in D_{3}^{2}$. Then by Lemma 5.2,

$$
\Gamma_{3}(\alpha) \cap \Gamma_{1}(x)=D_{2}^{3} .
$$

Take any $y \in D_{3}^{1}$ such that $x \sim y$ (see Fig. 11). Then

$$
\Gamma_{2}(y) \supseteq D_{2}^{3} .
$$

Claim $1 \quad \Gamma_{3}(x) \subseteq D_{2}^{1} \cup D_{1}^{2} \cup D_{1}^{3} \cup D_{0}^{3}, \Gamma_{3}(y) \subseteq D_{2}^{2} \cup D_{1}^{2} \cup D_{1}^{3} \cup D_{0}^{3}$.

Since $\Gamma_{1}(x) \supseteq D_{2}^{3}$ and the height $h=2$, we get

$$
\Gamma_{3}(x) \subseteq D_{2}^{1} \cup D_{2}^{2} \cup D_{1}^{2} \cup D_{1}^{3} \cup D_{0}^{3} .
$$




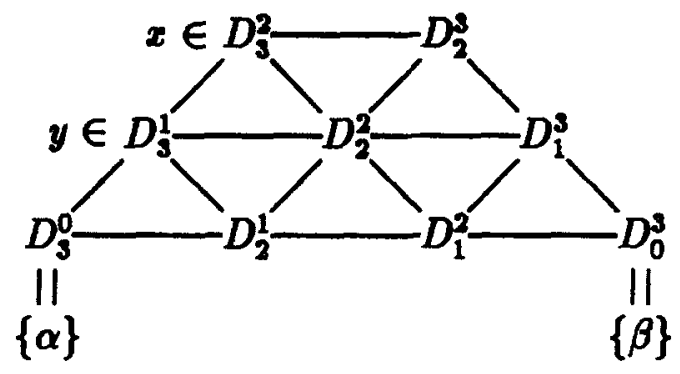

Figure 11 .

So we know

$$
\begin{aligned}
& \Gamma_{3}(x) \cap \Gamma_{2}(\beta)=\Gamma_{3}(x) \cap\left(D_{2}^{1} \cup D_{2}^{2}\right), \\
& \Gamma_{3}(x) \cap \Gamma_{1}(\alpha)=\Gamma_{3}(x) \cap D_{2}^{1} .
\end{aligned}
$$

Since $\partial(x, \alpha)=2$, by Lemma 5.2,

$$
\kappa_{2}=b_{2}=\left|\Gamma_{3}(x) \cap \Gamma_{1}(\alpha)\right|=\left|\Gamma_{3}(x) \cap D_{2}^{1}\right| .
$$

Since $\kappa_{2}=\left|\Gamma_{3}(x) \cap \Gamma_{2}(\beta)\right|=\left|\Gamma_{3}(x) \cap\left(D_{2}^{1} \cup D_{2}^{2}\right)\right|$, we get

$$
\Gamma_{3}(x) \cap D_{2}^{2}=\phi \text {. }
$$

Therefore

$$
\Gamma_{3}(x) \subseteq D_{2}^{1} \cup D_{1}^{2} \cup D_{1}^{3} \cup D_{0}^{3} .
$$

$\Gamma_{2}(y) \supseteq D_{2}^{3}$ and $y \sim \alpha$, hence we get

$$
\Gamma_{3}(y) \subseteq D_{2}^{2} \cup D_{1}^{2} \cup D_{1}^{3} \cup D_{0}^{3} .
$$

Claim $2 \quad \Gamma_{3}(y) \cap D_{1}^{2} \subseteq \Gamma_{3}(y) \cap \Gamma_{3}(x)$

Let $\gamma \in \Gamma_{3}(y) \cap D_{1}^{2}$. By Lemma 5.1, there is $\delta \in \Gamma_{3}(\alpha) \cap \Gamma_{3}(\gamma)$ such that $\delta \in D_{2}^{3}$. Then $x \sim \delta$. From Lemma 3.4(1), $\Gamma_{3}(\gamma)$ is geodetically closed. Since $y, \delta \in \Gamma_{3}(\gamma)$ with $\partial(y, \delta)=2$ and $y \sim x \sim \delta$, we get $x \in \Gamma_{3}(\gamma)$. Hence $\gamma \in \Gamma_{3}(y) \cap \Gamma_{3}(x)$.

Claim $3 \quad \Gamma_{3}(\alpha) \cap \Gamma_{3}(x) \subseteq \Gamma_{3}(y)$

Take any $\epsilon \in \Gamma_{3}(\alpha) \cap \Gamma_{3}(x)$. Since $\alpha, x \in \Gamma_{3}(\epsilon)$ with $\partial(\alpha, x)=2, \alpha \sim y \sim x$ and $\Gamma_{3}(\epsilon)$ is geodetically closed, we get $y \in \Gamma_{3}(\epsilon)$. Hence $\epsilon \in \Gamma_{3}(y)$.

Claim $4 \Gamma_{3}(y) \cap\left(D_{1}^{2} \cup D_{1}^{3} \cup D_{0}^{3}\right)=\Gamma_{3}(y) \cap\left(\Gamma_{3}(\alpha) \cup \Gamma_{3}(x)\right)$.

By Claim $2, \Gamma_{3}(y) \cap D_{1}^{2} \subseteq \Gamma_{3}(y) \cap \Gamma_{3}(x)$. Since $D_{1}^{3} \cup D_{0}^{3} \subseteq \Gamma_{3}(\alpha)$, $\Gamma_{3}(y) \cap\left(D_{1}^{3} \cup D_{0}^{3}\right) \subseteq \Gamma_{3}(y) \cap \Gamma_{3}(\alpha)$. Hence

$$
\Gamma_{3}(y) \cap\left(D_{1}^{2} \cup D_{1}^{3} \cup D_{0}^{3}\right) \subseteq \Gamma_{3}(y) \cap\left(\Gamma_{3}(\alpha) \cup \Gamma_{3}(x)\right) .
$$


On the other hand, take any $u \in \Gamma_{3}(y) \cap\left(\Gamma_{3}(\alpha) \cup \Gamma_{3}(x)\right)$. If $u \in \Gamma_{3}(y) \cap \Gamma_{3}(\alpha)$, then by Claim 1, $u \in \Gamma_{3}(y) \cap\left(D_{1}^{3} \cup D_{0}^{3}\right)$. If $u \in \Gamma_{3}(y) \cap \Gamma_{3}(x)$, then $u \in \Gamma_{3}(y) \cap\left(D_{1}^{2} \cup D_{1}^{3} \cup D_{0}^{3}\right)$. Therefore we get the claim.

Since $\alpha \sim y \sim x$ and $\partial(\alpha, x)=2$, by Claim 3,

$$
\begin{aligned}
& \left|\Gamma_{3}(y) \cap\left(\Gamma_{3}(\alpha) \cup \Gamma_{3}(x)\right)\right| \\
& \quad=\left|\Gamma_{3}(y) \cap \Gamma_{3}(\alpha)\right|+\left|\Gamma_{3}(y) \cap \Gamma_{3}(x)\right|-\left|\Gamma_{3}(\alpha) \cap \Gamma_{3}(x)\right| \\
& \quad=2 p_{33}^{1}-p_{33}^{2} .
\end{aligned}
$$

Since $\partial(y, \beta)=3$,

$$
\begin{aligned}
\kappa_{1} & =\left|\Gamma_{3}(y) \cap \Gamma_{1}(\beta)\right| \\
& =\left|\Gamma_{3}(y) \cap\left(D_{1}^{2} \cup D_{1}^{3}\right)\right| \\
& =\left|\Gamma_{3}(y) \cap\left(D_{1}^{2} \cup D_{1}^{3} \cup D_{0}^{3}\right)-\{\beta\}\right| .
\end{aligned}
$$

Hence by Claim 4, we get

$$
\kappa_{1}=2 p_{33}^{1}-p_{33}^{2}-1
$$

Lemma $5.4 p_{33}^{2}=1$.

Proof: By way of contradiction, suppose $p_{33}^{2} \geq 2$. Take $x \in D_{3}^{2}$. Since $\beta \in \Gamma_{3}(\alpha) \cap$ $\Gamma_{3}(x)$, there is $\gamma \in \Gamma_{3}(\alpha) \cap \Gamma_{3}(x)-\{\beta\}$. From Lemma 5.2, $\Gamma_{1}(x) \supseteq D_{2}^{3}$. Hence $\gamma \in D_{1}^{3}$ and $e\left(\gamma, D_{2}^{3}\right)=0$.

Claim $1 \quad \kappa_{1} \geq 2 \kappa_{2}-1$.

Since $b_{1}=e\left(\gamma, D_{2}^{2}\right)$, there is $\delta \in D_{2}^{2}$ such that $\gamma \sim \delta$. Suppose there is $y \in D_{2}^{3}$ such that $\delta \sim y$. As $e\left(\gamma, D_{2}^{3}\right)=0, \partial(\gamma, y)=2$. Since $\gamma, y \in \Gamma_{3}(\alpha)$ and $\Gamma_{3}(\alpha)$ is geodetically closed, we get $\delta \in \Gamma_{3}(\alpha)$. But this contradicts $\delta \in D_{2}^{2}$. So

$$
e\left(\delta, D_{2}^{3}\right)=0 \text {. }
$$

Hence

$$
b_{2}=e\left(\delta, D_{1}^{3}\right) \leq e\left(\delta, D_{1}^{3}\right)+e\left(\delta, D_{1}^{2}\right)=c_{2} .
$$

From Lemma 3.4(3) and 5.2,

$$
\kappa_{2}=b_{2} \leq c_{2}=\kappa_{1}-\kappa_{2}+1 .
$$

Since $e\left(\gamma, D_{2}^{3}\right)=0$, we can take $\epsilon \in D_{2}^{3}$ such that $\partial(\epsilon, \gamma)=2$ (see Fig. 12).

Claim $2 \Gamma_{3}(\gamma) \subseteq D_{3}^{0} \cup D_{3}^{1} \cup D_{3}^{2} \cup D_{2}^{2} \cup D_{2}^{1}, \Gamma_{3}(\epsilon) \subseteq D_{3}^{0} \cup D_{3}^{1} \cup D_{2}^{1} \cup D_{1}^{2}$.

By an argument similar to that in the Proof of Lemma 5.3, we have the claim.

Claim $3 \quad \Gamma_{3}(\gamma) \cap \Gamma_{3}(\epsilon) \cap D_{2}^{1}=\varnothing$. 


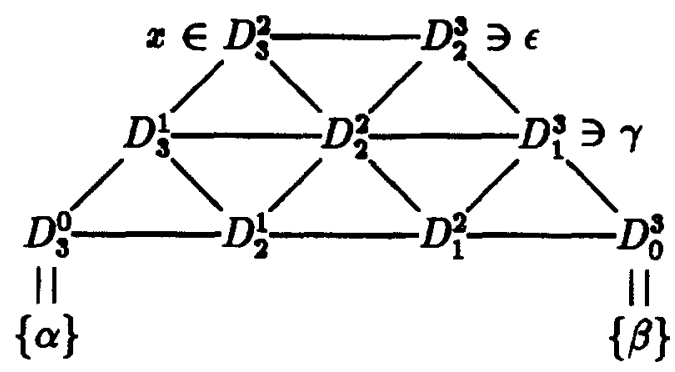

Figure 12.

Suppose there is $u \in \Gamma_{3}(\gamma) \cap \Gamma_{3}(\epsilon) \cap D_{2}^{1}$. Since $\gamma, \epsilon \in \Gamma_{3}(\alpha) \cap \Gamma_{3}(u)$ with $\partial(\epsilon, \gamma)=2$ and $\Gamma_{3}(\alpha) \cap \Gamma_{3}(u)$ is geodetically closed,

$$
\left(\Gamma_{1}(\gamma) \cap \Gamma_{1}(\epsilon)\right) \cup\{\gamma\} \cup\{\epsilon\} \subseteq \Gamma_{3}(\alpha) \cap \Gamma_{3}(u)
$$

Since $e\left(\gamma, D_{2}^{3}\right)=0$,

$$
\left(\Gamma_{1}(\gamma) \cap \Gamma_{1}(\epsilon)\right) \cup\{\gamma\} \subseteq \Gamma_{3}(u) \cap D_{1}^{3} \subseteq \Gamma_{3}(u) \cap \Gamma_{1}(\beta)
$$

As $\partial(\gamma, \epsilon)=\partial(u, \beta)=2$, we get

$$
c_{2}+1 \leq b_{2}
$$

From Lemma 3.4(3) and 5.2,

$$
\kappa_{1}-\kappa_{2}+2 \leq \kappa_{2}
$$

This contradicts Claim 1.

Claim $4 p_{33}^{2}+c_{2}+1 \leq p_{33}^{1}$.

From Claim 2 and 3,

$$
\Gamma_{3}(\gamma) \cap \Gamma_{3}(\epsilon) \subseteq \Gamma_{3}(\beta) \cap \Gamma_{3}(\gamma)
$$

Since $\alpha, x \in \Gamma_{3}(\beta) \cap \Gamma_{3}(\gamma)$ with $\partial(\alpha, x)=2$ and $\Gamma_{3}(\beta) \cap \Gamma_{3}(\gamma)$ is geodetically closed,

$$
\left(\Gamma_{1}(\alpha) \cap \Gamma_{1}(x)\right) \cup\{x\} \cup\{\alpha\} \subseteq \Gamma_{3}(\beta) \cap \Gamma_{3}(\gamma) .
$$

As $\alpha \in \Gamma_{3}(\gamma) \cap \Gamma_{3}(\epsilon)$,

$$
\left(\Gamma_{3}(\gamma) \cap \Gamma_{3}(\epsilon)\right) \cup\left(\left(\Gamma_{1}(\alpha) \cap \Gamma_{1}(x)\right) \cup\{x\}\right) \subseteq \Gamma_{3}(\beta) \cap \Gamma_{3}(\gamma) .
$$

By Lemma 5.2, $x \sim \epsilon$. So

$$
\Gamma_{3}(\epsilon) \cap\left(\left(\Gamma_{1}(\alpha) \cap \Gamma_{1}(x)\right) \cup\{x\}\right)=\emptyset
$$

So we get

$$
\left(\Gamma_{3}(\gamma) \cap \Gamma_{3}(\epsilon)\right) \cap\left(\left(\Gamma_{1}(\alpha) \cap \Gamma_{1}(x)\right) \cup\{x\}\right)=\emptyset
$$


Therefore as $\partial(\gamma, \epsilon)=\partial(\alpha, x)=2$ and $\beta \sim \gamma$,

$$
\begin{gathered}
\left|\left(\Gamma_{3}(\gamma) \cap \Gamma_{3}(\epsilon)\right) \cup\left(\left(\Gamma_{1}(\alpha) \cap \Gamma_{1}(x)\right) \cup\{x\}\right)\right| \leq\left|\Gamma_{3}(\beta) \cap \Gamma_{3}(\gamma)\right|, \\
p_{33}^{2}+c_{2}+1 \leq p_{33}^{1} .
\end{gathered}
$$

From Claim 4 and Lemma 5.3,

$$
\begin{gathered}
2\left(p_{33}^{2}+c_{2}+1\right) \leq \kappa_{1}+p_{33}^{2}+1 \\
2\left(p_{33}^{2}+\kappa_{1}-\kappa_{2}+2\right) \leq \kappa_{1}+p_{33}^{2}+1 \\
p_{33}^{2} \leq-\kappa_{1}+2 \kappa_{2}-3
\end{gathered}
$$

From Claim 1, we get

$$
p_{33}^{2} \leq-2
$$

This is impossible. Hence we get

$$
p_{33}^{2}=1
$$

Lemma 5.5 The following hold.

(1) $2 p_{33}^{1}=\kappa_{1}+2$,

(2) $p_{33}^{1}\left(\kappa_{2}^{2}+\kappa_{1}\right)=\kappa_{1}\left(1+\kappa_{1}+\kappa_{2}\right)$.

\section{Proof:}

(1) It is clear from Lemma 5.3 and 5.4.

(2) It follows from Lemma 5.4 that

$$
k_{2}=p_{33}^{2} k_{2}=p_{32}^{3} k_{3}=\kappa_{2}\left(1+\kappa_{1}+\kappa_{2}\right)
$$

Since $c_{3}\left(1+\kappa_{1}+\kappa_{2}\right)=c_{3} k_{3}=b_{2} k_{2}=\kappa_{2}^{2}\left(1+\kappa_{1}+\kappa_{2}\right)$,

$$
c_{3}=\kappa_{2}^{2} \text {. }
$$

Hence

$$
k=c_{3}+a_{3}=\kappa_{2}^{2}+\kappa_{1} .
$$

Since $p_{33}^{1} k=p_{31}^{3} k_{3}$, we get

$$
p_{33}^{1}\left(\kappa_{2}^{2}+\kappa_{1}\right)=\kappa_{1}\left(1+\kappa_{1}+\kappa_{2}\right)
$$

Proof of Theorem 1.1. From Lemma 5.5,

$$
\begin{aligned}
& \left(\kappa_{1}+2\right)\left(\kappa_{2}^{2}+\kappa_{1}\right)=2 \kappa_{1}\left(1+\kappa_{1}+\kappa_{2}\right) . \\
& \left(\kappa_{1}+2\right) \kappa_{2}^{2}-2 \kappa_{1} \kappa_{2}-\kappa_{1}^{2}=0 .
\end{aligned}
$$

Hence we get

$$
\kappa_{2}=\frac{\kappa_{1}+\kappa_{1} \sqrt{\kappa_{1}+3}}{\kappa_{1}+2}
$$


Since $\kappa_{2}$ is a positive integer, by Lemma $3.4(2), \sqrt{\kappa_{1}+3}$ is a positive integer at least 3 . Let $n=\sqrt{\kappa_{1}+3}$. Then

$$
\kappa_{2}=\frac{\left(n^{2}-3\right)(n+1)}{n^{2}-1}=n+1-\frac{2}{n-1} .
$$

Hence we get $n=3$ and

$$
\kappa_{1}=6, \quad \kappa_{2}=3 .
$$

Therefore we know all the intersection numbers of $\Gamma$ and they are the same as those of $J(8,3)$. By the uniqueness (see [7] and [10]), we get

$$
\Gamma \simeq J(8,3) \text {. }
$$

\section{Appendix}

The next theorem was proved by A. Hiraki and H. Suzuki.

Theorem Let $\Delta$ be a complete multipartite graph $K_{\tau \times s}$ with $\tau \geq 2, s \geq 2$. Then there is no distance-regular graph $\Gamma$ with diameter $d \geq 3$ such that $\Gamma_{d}(\alpha) \simeq \Delta$ for every vertex $\alpha$ in $\Gamma$.

Proof: The intersection array of $\Delta$ is as follows.

$$
\iota(\Delta)=\left\{\begin{array}{ccc}
* & 1 & (\tau-1) s \\
0 & (\tau-2) s & 0 \\
(\tau-1) s & s-1 & *
\end{array}\right\}
$$

Suppose there exists a graph $\Gamma$ satisfying the hypothesis. Take any $\alpha, \beta \in \Gamma$ with $\partial(\alpha, \beta)=$ $d$. Then $p_{d 1}^{d}=\left|\Delta_{1}(\beta)\right|=(\tau-1) s, p_{d 2}^{d}=\left|\Delta_{2}(\beta)\right|=s-1$ and $k_{d}=|\Delta|=\tau s$. By an argument similar to that in Section 3, the intersection diagram becomes as in Fig. 13.

Since $\Delta_{2}(\beta)$ is a coclique, $D_{2}^{d}$ is a coclique. For any $x \in D_{1}^{d}$ and $y \in D_{2}^{d}$, we know $e\left(x, D_{1}^{d}\right)=(\tau-2) s$ and $e\left(y, D_{1}^{d}\right)=(\tau-1) s$.

Claim 1 For every $\alpha, \gamma \in \Gamma$ with $\partial(\alpha, \gamma)=d-1, \Gamma_{d}(\alpha) \cap \Gamma_{1}(\gamma)$ is a coclique.

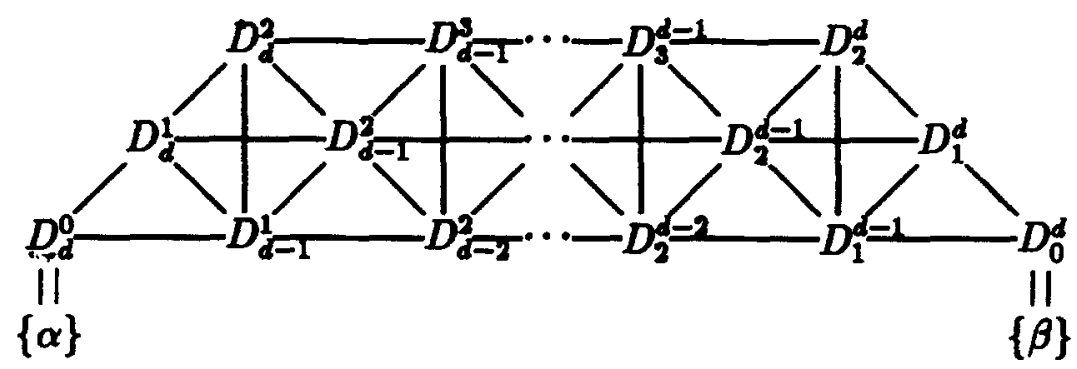




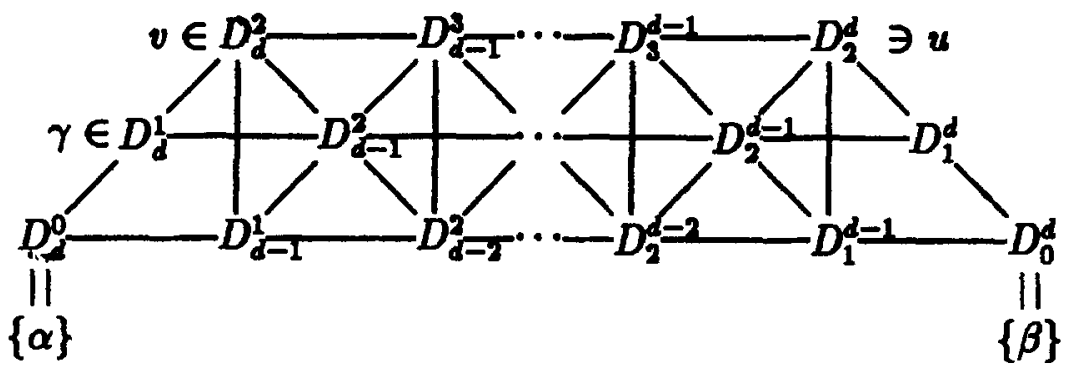

Figure 14.

Since $p_{d 3}^{d-1} \neq 0$, we can take $\beta \in \Gamma_{d}(\alpha) \cap \Gamma_{3}(\gamma)$. Then $\gamma \in D_{3}^{d-1}$. As $\Gamma_{d}(\alpha) \cap \Gamma_{1}(\gamma) \subseteq$ $D_{2}^{d}$ and $D_{2}^{d}$ is a coclique, we get the claim.

Claim $2 e\left(D_{1}^{d-1}, D_{1}^{d}\right)=0, a_{1}=(\tau-2) s$.

Suppose there is an edge $\gamma \sim \delta$ such that $\gamma \in D_{1}^{d-1}, \delta \in D_{1}^{d}$. Then $\Gamma_{d}(\alpha) \cap \Gamma_{1}(\gamma)$ contains an edge $\beta \sim \delta$, which contradicts Claim 1 .

For any $x \in D_{1}^{d}$,

$$
a_{1}=e\left(x, D_{1}^{d}\right)=(\tau-2) s
$$

Claim 3 For every edge $\alpha \sim \gamma, \Gamma_{d}(\alpha) \cap \Gamma_{d}(\gamma)$ is a clique. $p_{d d}^{1}=\tau, k=(\tau-1) s^{2}$.

Take $\beta, \delta \in \Gamma_{d}(\alpha) \cap \Gamma_{d}(\gamma)$. Then $\gamma \in D_{d}^{1}$. For any $u \in D_{2}^{d}$, there is $v \in D_{d}^{2}$ such that $\partial(u, v)=d-2$ (see Fig. 14).

Since $\gamma \sim v, \partial(\gamma, u)=d-1$. So $\delta \in D_{1}^{d}$. Hence

$$
\beta \sim \delta
$$

Therefore $\Gamma_{d}(\alpha) \cap \Gamma_{d}(\gamma)$ is a clique. Since the size of the maximal cliques of $\Gamma_{d}(\alpha) \simeq \Delta$ is $\tau$,

$$
p_{d d}^{1} \leq \tau
$$

Suppose $p_{d d}^{1} \leq \tau-1$, then

$$
k=\frac{k_{d} p_{d 1}^{d}}{p_{d d}^{1}} \geq \tau s^{2}>\tau s(s-1) .
$$

Since $p_{d d}^{2} \geq 1$,

$$
k_{2}=\frac{k_{d} p_{d 2}^{d}}{p_{d d}^{2}} \leq \tau s(s-1) .
$$

So $k>k_{2}$, which is impossible (see Lemma 5.1.2 of [5]). Hence we get $p_{d d}^{1}=\tau$.

Claim 4 For every $\alpha \in \Gamma$ and every edge $\beta \sim \gamma$ in $\Gamma_{d}(\alpha), \Gamma_{1}(\beta) \cap \Gamma_{1}(\gamma) \subseteq \Gamma_{d}(\alpha)$. 
Since $\gamma \in D_{1}^{d}$, the claim follows from Claim 2 .

Claim $5 \tau=2, p_{d d}^{1}=2, a_{1}=0, k=s^{2}, b_{1}=s^{2}-1, k_{2} \leq 2 s(s-1)$.

Since $\tau \geq 2$, there is an edge $\beta \sim \delta$ in $\Gamma_{d}(\alpha) \cap \Gamma_{d}(\gamma)$ for $\alpha \sim \gamma$. From Claim 4,

$$
\left(\Gamma_{1}(\beta) \cap \Gamma_{1}(\delta)\right) \cup\{\beta\} \cup\{\delta\} \subseteq \Gamma_{d}(\alpha) \cap \Gamma_{d}(\gamma) .
$$

In this case

$$
\begin{aligned}
a_{1}+2 & \leq p_{d d}^{1}, \\
(\tau-2) s+2 & \leq \tau .
\end{aligned}
$$

Since $\tau \geq 2$ and $s \geq 2$, we have

$$
\tau=2
$$

So we get the claim.

Claim $6 d=3$.

Since $k b_{1}=k_{2} c_{2}$, Claim 5 implies that

$$
\frac{s^{2}\left(s^{2}-1\right)}{c_{2}}=k_{2} \leq 2 s(s-1)
$$

So

$$
k=s^{2}<s(s+1) \leq 2 c_{2} .
$$

Since $b_{2} \leq b_{2}+a_{2}=k-c_{2}<c_{2}$, we get $d=3$.

By Claim 6, the intersection diagram is as in Fig. 15.

By counting $e\left(D_{2}^{3}, D_{1}^{2}\right)$,

$$
\begin{aligned}
\left|D_{1}^{2}\right|\left(b_{2}-1\right) & =\left|D_{2}^{3}\right|\left(c_{2}-s\right), \\
\left(s^{2}-s\right)\left(b_{2}-1\right) & =(s-1)\left(c_{2}-s\right), \\
s b_{2} & =c_{2} .
\end{aligned}
$$

Since $k b_{1} b_{2}=k_{3} c_{3} c_{2}$,

$$
\begin{aligned}
s^{2}\left(s^{2}-1\right) b_{2} & =2 s\left(s^{2}-s\right) c_{2}, \\
(s+1) b_{2} & =2 c_{2} .
\end{aligned}
$$

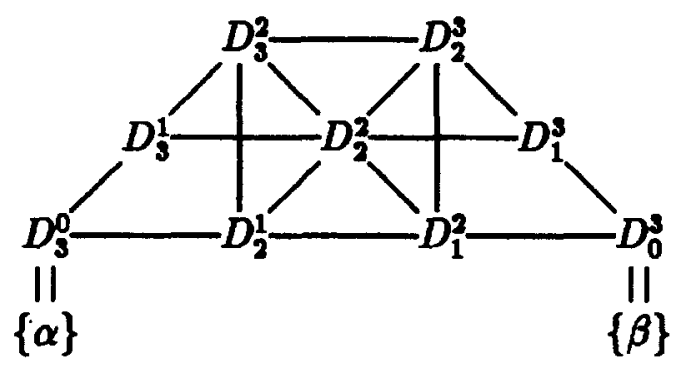

Figure 15. 
Hence we get

$$
(s+1) b_{2}=2 s b_{2}
$$

Since $s \geq 2$, this is impossible. Therefore we get the assertion.

\section{Acknowledgment}

I would like to thank Prof. H. Suzuki for his advice on this paper. Some of the proofs in this paper were shortened thorough the discussions with him.

\section{References}

1. E. Bannai and T. Ito, Algebraic Combinatorics I, Benjamin-Cummings, California, 1984.

2. E. Bannai and T. Ito, "Current researches on algebraic combinatorics," Graphs and Combin. 2 (1986), 287308.

3. N.L. Biggs, Algebraic Graph Theory, Cambridge University Press, Cambridge, 1974.

4. A. Boshier and K. Nomura, "A remark on the intersection arrays of distance regular graphs," J. Combin. Theory Ser. B. 44 (1988), 147-153.

5. A.E. Brouwer, A.M. Cohen, and A. Neumaier, Distance-Regular Graphs, Springer, Berlin-Heidelberg, 1989.

6. A.D Gardiner, C.D. Godsil, A.D. Hensel, and Gordon F. Royle, "Second neighbourhoods of strongly regular graphs," Discrete Math. 103 (1992), 161-170.

7. A. Neumaier, "Characterization of a class of distance-regular graphs," J. Reine Angew. Math. 357 (1985), $182-192$.

8. H. Suzuki, "Bounding the diameter of a distance regular graph by a function of $k_{d}$," Graphs and Combin. 7 (1991), 363-375.

9. H. Suzuki, "Bounding the diameter of a distance regular graph by a function of $k_{d}$, II," J. Algebra 169 (1994), 713-750.

10. P. Terwilliger, "The Johnson graph $J(d, r)$ is unique if $(d, r) \neq(2,8), "$ Discrete Math. 58 (1986), 175-189. 